\title{
EFECTO DEL MANEJO SILVÍCOLA Y CLASE DE COPA SOBRE LA DENSIDAD BÁSICA DE Eucalyptus nitens
}

\author{
Eduardo Rodrigo Navarrete Espinoza ${ }^{1}$, Ximena Figueroa Cardenas ${ }^{2}$, Pablo Andrés Novoa Barra ${ }^{1}$, \\ Miguel Angel Espinosa Bancalari ${ }^{3}$ \\ ${ }^{1}$ Ing., M.Sc., Universidad de Concepción, Depto. Forestal, Grupo de Invest. GESE, Los Ángeles, Chile - ednavarr@udec.cl - \\ panovoa@udec.cl \\ ${ }^{2}$ Ing., Universidad de Concepción, Depto Forestal, Los Ángeles, Chile - jefiguer@udec.cl \\ ${ }^{3}$ Ing., Ph.D., Universidad de Concepción, Facultad de Ciencias Forestales, Concepción, Chile - mespinos@udec.cl
}

Recebido para publicação: 22/11/2007 - Aceito para publicação: 04/09/2008

\begin{abstract}
Resumen
Para analizar el efecto del manejo silvícola sobre la variación de la densidad básica de la madera de Eucalyptus nitens, se estudió una plantación en el sector precordillerano andino de la VIII región en Chile. El estudio se realizó en un rodal de 10 años, intervenido a los 2 años y medio de establecido, evaluándose distintas intensidades de poda y densidades de raleo. En cada tratamiento definido, se estudió también el efecto de la clase de copa sobre el comportamiento de la densidad básica. Se muestreó un total de 36 árboles, obteniéndose de cada uno, siete discos a diferentes alturas del fuste, calculándose finalmente su densidad básica. Los resultados determinaron que el raleo afectó la densidad básica, presentando los rodales sin intervención, una densidad básica promedio menor que aquellos intervenidos; en cambio en aquellas unidades muestrales con poda, se presentaron los menores valores promedios para esta variable. La clase de copa dominante presentó una densidad básica superior a la codominante y suprimida respectivamente. En cuanto al comportamiento de la densidad de la madera en función de la altura de muestreo en el fuste, ésta tendió a aumentar desde la base al ápice, aunque presentó una leve disminución en las primeras secciones del árbol.

Palabras-clave: Eucalyptus nitens; poda; raleo; densidad de madera.
\end{abstract}

\begin{abstract}
Effect of silvicultural management and type of crown on basic density of Eucalyptus nitens. This research was curried out in the $8^{\text {th }}$ region andean lower part in Chile and aim to analyze the effect of silvicultural management about Eucalyptus nitens basic density variation. The study was based on a 10 year-old stand which was pruned and thinned at the age of 30 months, evaluating different pruning intensity and thinning density. It was also studied, in every defined treatment, the effect produced by different types of crown on the basic density behavior. The total sample consisted on 36 trees from which 7 different discs were obtained and every one had different: trunk height, being finally calculated their basic density. The results determined that thinning affected the basic density and nonintervened stands presented a minor average basic density than those intervened; on the other hand, those plots with pruning presented the lowest average for this variable. The dominant crown presented a higher basic density than the co-dominant and suppressed one respectively. Concerning wood density in relation to the sample trunk height, this tended to increase from the buton to the tip even though it presented a small decrease in the first sections of the tree.

Keywords: Eucalyptus nitens; pruning; thinning; wood density.
\end{abstract}

\section{INTRODUCCIÓN}

Los Eucalyptus adquieren cada vez más importancia en el mundo, siendo prueba de ello la existencia de más de 15 millones de hectáreas plantadas en más de 100 países. La gran superficie plantada con Eucalyptus se debe fundamentalmente a los múltiples usos que se pueden dar a sus especies, entre las que se cuentan: madera aserrada, paneles, aglomerados, postes, chapas, pulpa, entre otros. El éxito de este género a nivel mundial se debe además, a la gran adaptabilidad que posee, lo que ha permitido su desarrollo en una amplia gama de ambientes ecológicos, desde desérticos hasta templados-fríos. Ello se 
une a una excelente combinación de: rápido crecimiento, peso específico y producción volumétrica, que lo define como uno de los géneros más difundidos en el mundo (HERNÁNDEZ, 2002).

En Chile se han establecido más de 400 mil hectáreas de plantaciones de varias especies de Eucalyptus, siendo Eucalyptus globulus la principal (80\%) y preferida por la industria de la celulosa, seguido por Eucalyptus nitens (20\%) plantado en zonas de frío, donde E. globulus no prospera, y que no sólo presenta posibilidades de transformación como madera pulpable, sino que exhibe un gran potencial para la producción de madera sólida de alto valor (CABRERA, 2001). Este hecho ha sido detectado en Australia hace ya 10 años, donde E. nitens tiene un importante potencial para la producción de madera aserrada y chapas, por lo que se ha convertido en una de las principales especies plantadas en Tasmania (VALENCIA; CABRERA, 2003).

La creciente demanda por madera de E. globulus y E. nitens en diferentes procesos industriales, tanto para el mercado nacional como internacional, requiere de un conocimiento acabado de la calidad de la materia prima para obtener productos de mejor estándar y mayor valor agregado. Hoy en día existe un notable incremento en la intensidad de manejo de rodales, con el objetivo de aumentar la producción maderera. Esto implica una mayor demanda por información respecto a la calidad esperada de madera, dadas diferentes prescripciones de planificación silvicultural. Los simuladores de crecimiento, tal como el EUCASIM, son una valiosa herramienta para proveer de información sobre la cantidad total de volumen maderero a obtener bajo diferentes escenarios silviculturales; sin embargo, estos no predicen la calidad de la madera bajo distintas opciones de manejo silvícola.

Históricamente, el manejo silvícola del recurso bosque se ha orientado principalmente al aumento de la tasa de crecimiento de las plantaciones. Como consecuencia de esto, existe una completa desconexión entre la aplicación de silvicultura y los requerimientos de las empresas de transformación de la madera. El conocimiento de la anatomía, propiedades físicas, químicas y mecánicas de la madera de las especies forestales, son indispensables si se quiere contar con una base sólida que permita determinar su utilización adecuada (VALENCIA; LÓPEZ, 1999). Una de las principales características de la madera es su heterogeneidad, por ende, el conocimiento detallado de sus propiedades y variabilidad, son importantes para prever el comportamiento de los productos elaborados (DANIEL et al., 1982). De las características de la madera, la densidad básica es una de las más importantes, ya que existe una estrecha relación entre esta última y las características físico-mecánicas de la madera. Una mayor densidad implica un aumento en la resistencia en construcción, mayor rendimiento en la elaboración de pulpa para papel y mayor valor calorífico al utilizar la madera como combustible (KOLLMAN, 1959). La densidad de la madera presenta gran variabilidad, reconociéndose la variación que existe entre sitios, rodales, especies y árboles, información que se utiliza por ejemplo, en programas de mejoramiento genético forestal (ZOBEL y TALBERT, 1988). En general, las características de la madera de una especie depende de una serie de factores, entre ellos se destacan la edad, clima, suelo y silvicultura (BAMBER; BURLEY, 1983); además de la ubicación geográfica, especie y sitio (HAYGREEN; BOWYER, 1996).

La importancia de la densidad básica se debe a que puede ser utilizada como elemento de juicio en la selección y/o clasificación de maderas (CAMPOS et al., 1990), considerándose un buen indicador de la calidad en varios usos (ROZENBERG et al., 2001). Se ha determinado además, que es uno de los índices más importantes de resistencia en la madera, por lo que ha sido positivamente correlacionada con propiedades tales como el módulo de ruptura (MOR) y el módulo de elasticidad (MOE) (FERNÁNDEZGOLFÍN; DIEZ, 1994); teniendo además, una considerable influencia en la trabajabilidad, conversión y propiedades acústicas (USTA; HALE, 2002). Greaves; Borralho (1996) determinaron que la densidad básica es determinante en los cálculos de costos de los procesos de digestión y obtención de pulpa kraft.

Dentro de la caracterización de la variabilidad de la densidad básica, se puede decir que por su relación con la calidad de los productos finales, la densidad y su variabilidad son de gran interés para los aserraderos e industrias papeleras (TIAN et al., 1995).

El objetivo general de este estudio es identificar los diferentes factores que influyen en la variación de la densidad básica de la madera de E. nitens. Los objetivos específicos propuestos son analizar la influencia del manejo silvícola, específicamente distintas intensidades de poda y raleo, en la densidad básica de la madera de E. nitens; además de describir los patrones de comportamiento longitudinal de la densidad básica en el fuste, para las clases de copa dominante, codominante-intermedia y suprimida. 


\section{MATERIALES Y MÉTODOS}

\section{Área de estudio}

El ensayo se ubica en el predio Villucura, comuna de Santa Bárbara, de propiedad de la Universidad de Concepción, en el sector precordillerano andino de la VIII región en Chile $\left(37^{\circ} 35^{\prime} \mathrm{S}\right.$ y $72^{\circ} 00^{\prime} \mathrm{W}$ ), aproximadamente a $460 \mathrm{~m} . \mathrm{s} . n$.m. Presenta una topografía ondulada a quebrada, con un suelo de capacidad de uso IV perteneciente a la serie Santa Bárbara, esto es principalmente textura arcillosa, compactada y con algunos sectores erosionados (CARRASCO et al., 1989). El clima es templado, presentando una temperatura y precipitación media anual de $13,8{ }^{\circ} \mathrm{C}$ y $1.389 \mathrm{~mm}$, respectivamente (ITURRIA, 1997).

\section{Descripción del lugar de estudio}

El estudio se realizó en una plantación de E. nitens de diez años de edad, la cual fue intervenida a los dos años y medio de establecida, con el objetivo de evaluar la influencia del manejo silvícola en el crecimiento y dominancia de la especie. Los tratamientos aplicados a la plantación fueron tres intensidades de poda, acompañadas por tres densidades de raleo residual. De la combinación de los niveles de poda y raleo, se definieron un total de nueve tratamientos a aplicar (Tabla 1), con tres réplicas cada uno (parcelas), lo que constituyó un total de 27 unidades muestrales. Cada unidad muestral contó con una superficie de $252 \mathrm{~m}^{2}(21 \mathrm{~m} \times 12 \mathrm{~m})$.

Tabla 1. Descripción de los tratamientos silvícolas aplicados a la plantación de E. nitens.

Table 1. Description of the applied silvicultural treatments at E. nitens plantation.

\begin{tabular}{lccc}
\hline Poda $(\mathbf{P})$ & \multicolumn{3}{c}{ Raleo (R) } \\
\cline { 2 - 4 } & $\mathbf{R}_{\mathbf{1 6 0 0}}$ & $\mathbf{R}_{\mathbf{1 2 0 0}}$ & $\mathbf{R}_{\mathbf{8 0 0}}$ \\
\hline $\mathrm{P}_{0 \%}$ & $\mathrm{P}_{0 \%} \mathrm{R}_{1600}$ & $\mathrm{P}_{0 \%} \mathrm{R}_{1200}$ & $\mathrm{P}_{0 \%} \mathrm{R}_{800}$ \\
$\mathrm{P}_{40 \%}$ & $\mathrm{P}_{40 \%}{ }^{(*)} \mathrm{R}_{1600}$ & $\mathrm{P}_{40 \%} \mathrm{R}_{1200}$ & $\mathrm{P}_{40 \%}{ }^{*} \mathrm{R}_{800}$ \\
$\mathrm{P}_{60 \%}$ & $\mathrm{P}_{60 \%} \mathrm{R}_{1600}$ & $\mathrm{P}_{60 \%} \mathrm{R}_{1200}$ & $\mathrm{P}_{60 \%} \mathrm{R}_{800}$ \\
\hline
\end{tabular}

Donde:

Poda (porcentaje de altura podada)

$\mathrm{P}_{0 \%}$ : sin poda (testigo)

$\mathrm{P}_{40} \%$ : poda hasta un $40 \%$ de la altura total

$\mathrm{P}_{60 \%}$ : poda hasta un $60 \%$ de la altura total
Raleo (densidad residual)

$\mathrm{R}_{1600}: 1600$ árb/ha (testigo)

$\mathrm{R}_{1200}: 1200$ árb/ha

$\mathrm{R}_{800}: 800$ árb/ha

\section{Diseño experimental del estudio}

Para identificar el efecto de las intervenciones silviculturales en la variación de la densidad básica de la madera de E. nitens, se evaluaron los siguientes niveles de poda y raleo, así como sus respectivas combinaciones: $\mathrm{P}_{0 \%}, \mathrm{P}_{60} \%, \mathrm{R}_{1600}$ y $\mathrm{R}_{800}$ (Tabla $1(*)$ ). La elección de estos tratamientos se basa en los resultados de crecimiento obtenidos en el estudio denominado "Efecto del manejo temprano, en el crecimiento y dominancia en rodales jóvenes de Eucalyptus globulus y Eucalyptus nitens en un suelo precordillerano de la VIII Región” (BRITO, 2003), en el cual se determinó la existencia de diferencias significativas en crecimiento diametral, entre los tratamientos mencionados $(*)$.

Los tratamientos a estudiar quedaron definidos por la combinación de todos los niveles de los factores que se detallan a continuación:

\section{A: Factor poda:}

Niveles:

$\mathrm{a}_{0}: \sin$ poda

$a_{1}: 60 \%$ de la altura total

\section{B: Factor raleo:}

Niveles:

$\mathrm{b}_{0}: 1600$ árb/ha (densidad inicial)

$\mathrm{b}_{1}: 800$ árb/ha 
C: Factor clase de copa:

Niveles:

$\mathrm{c}_{0}$ : dominante

$\mathrm{c}_{1}$ : codominante e intermedio

$\mathrm{c}_{2}$ : suprimido

\section{D: Factor altura relativa}

Niveles

$\mathrm{d}_{1}$ : Diámetro altura tocón (DAT)

$\mathrm{d}_{2}$ : Diámetro altura de pecho (DAP)

$\mathrm{d}_{3}$ : Inicio copa muerta (ICM)

$\mathrm{d}_{4}$ : Mitad altura del árbol (Mitad)

$\mathrm{d}_{5}$ : Antes de copa viva (ACV)

$\mathrm{d}_{6}$ : Después de copa viva (DCV)

$\mathrm{d}_{7}$ : Diámetro límite de utilización (DLU $6 \mathrm{~cm}$ )

Como resultado de la combinación de todos los niveles para los cuatro factores, se determinaron 84 tratamientos, con tres réplicas por tratamiento (36 árboles $\times 7$ rodelas/árbol $=252$ rodelas).

\section{Caracterización de las condiciones de rodal y árboles individuales}

Estimación de parámetros y medición de variables.

1. Características dasométricas (registro): diámetro y altura promedios, área basal por ha, número de árboles por ha.

2. Características dendrométricas (árboles muestra): altura total, diámetro a la altura de pecho (DAP), clase de copa, altura comienzo de copa viva.

Posterior al registro de las variables a nivel de árbol individual (árboles muestra) se procedió a voltear y extraer rodelas de $3 \mathrm{~cm}$ de espesor, a diferentes alturas en el fuste: Diámetro altura tocón (DAT), diámetro a la altura de pecho (DAP), inicio de copa muerta (ICM), mitad de la altura del árbol (50\% de la altura total), antes de copa viva (ACV), después de copa viva (DCV), además del diámetro límite de utilización (DLU $6 \mathrm{~cm}$ ).

\section{Actividades de laboratorio}

Cada rodela fue etiquetada, para posteriormente medir su diámetro. Cada una de las muestras fue pesada y medida en verde, según NCh 176/1 y 176/2 (INN, 1984, 1986). Posteriormente, éstas fueron secadas en horno a $85^{\circ} \mathrm{C}$ hasta la obtención de peso anhidro. Calculándose finalmente la densidad básica de las muestras de acuerdo a la relación:

$$
\text { Densidad }=\frac{p m s}{v}(1)
$$

Donde:

pms $=$ peso seco de la muestra $(\mathrm{g})$

$\mathrm{v}=$ volumen saturado de la muestra $\left(\mathrm{cm}^{3}\right)$

\section{Análisis de la información.}

El efecto de las distintas fuentes de variación (características de manejo, rodal y árbol) sobre la densidad básica de la madera y sus interacciones fue evaluado a través de análisis de varianza (ANDEVA) por medio de prueba F, siendo las diferencias identificadas por medio del test de Tukey, con un nivel de significancia de 0,05 (MONTGOMERY, 1991).

\section{RESULTADOS Y DISCUSIÓN}

\section{Situación actual de los tratamientos estudiados}

En la tabla 2, se observa que el tratamiento $\mathrm{P}_{0} \mathrm{R}_{800}$ presenta los mayores valores absolutos en DAP promedio, área basal y altura media, aunque no difieren estadísticamente de los demás tratamientos. Lo anterior puede explicarse debido a que el rodal fue intervenido silviculturalmente a temprana edad, y si bien los tratamientos presentaron diferencias significativas hasta los cuatro años de intervenidos (BRITO, 2003), este efecto tendió a anularse, como consecuencia de la ausencia de una segunda intervención. Según Balloni (1983) a medida que el espaciamiento aumenta, dando origen a densidades intermedias, la altura media tiende a aumentar, disminuyendo nuevamente cuando los espacios son muy amplios. Este hecho se debe a que la disminución del espaciamiento aumenta la competencia, lo cual 
produce un mayor número de árboles suprimidos, lo que explica la disminución del crecimiento medio del rodal. Esta tendencia no sólo se encuentra en los eucaliptos, sino que en la mayoría de las especies forestales de rápido crecimiento (PRADO; BARROS, 1989).

Tabla 2. Caracterización de las variables estudiadas por tratamientos.

Table 2. Characterization of the studiet variables by treatment.

\begin{tabular}{lcccc}
\hline \multirow{2}{*}{ Variable } & \multicolumn{4}{c}{ Tratamiento } \\
\cline { 2 - 4 } & $\mathbf{P}_{\mathbf{0}} \mathbf{R}_{\mathbf{8 0 0}}$ & $\mathbf{P}_{\mathbf{0}} \mathbf{R}_{\mathbf{1 6 0 0}}$ & $\mathbf{P}_{\mathbf{6 0}} \mathbf{R}_{\mathbf{8 0 0}}$ & $\mathbf{P}_{\mathbf{6 0}} \mathbf{R}_{\mathbf{1 6 0 0}}$ \\
\hline DAP Promedio $(\mathrm{cm})$ & $22,2 \mathrm{a}$ & $19,1 \mathrm{a}$ & $21,0 \mathrm{a}$ & $20,8 \mathrm{a}$ \\
Área Basal $(\mathrm{G})\left(\mathrm{m}^{2} / \mathrm{ha}\right)$ & $1,652 \mathrm{a}$ & $1,239 \mathrm{a}$ & $1,442 \mathrm{a}$ & $1,497 \mathrm{a}$ \\
Altura promedio $(\mathrm{m})$ & $32,3 \mathrm{a}$ & $26,3 \mathrm{a}$ & $26,3 \mathrm{a}$ & $28,3 \mathrm{a}$ \\
\hline En filas, les
\end{tabular}

En filas, letras iguales no difieren estadísticamente $(\mathrm{p}=0,05)$

Análisis de los diferentes factores en estudio.

En la tabla 3 se presenta el análisis de varianza, donde se observa el efecto de cada fuente de variación estudiada, respecto al comportamiento de la densidad básica de la madera.

Tabla 3. Análisis de varianza para los distintos factores estudiados.

Table 3. Analysis of variance of factors studied.

\begin{tabular}{lccc}
\hline Fuente de variación & g.l & F & Valor-p \\
\hline Poda $(\mathrm{P})$ & 1 & 2,87 & 0,092 \\
Raleo (R) & 1 & 1,97 & 0,163 \\
Clase de copa (C) & 2 & 13,24 & $<0,001$ \\
Altura relativa (H) & 6 & 33,27 & $<0,001$ \\
$\mathrm{P} \times \mathrm{R}$ & 1 & 6,77 & 0,010 \\
$\mathrm{P} \times \mathrm{C}$ & 2 & 0,87 & 0,420 \\
$\mathrm{R} \times \mathrm{C}$ & 2 & 1,38 & 0,255 \\
$\mathrm{P} \times \mathrm{H}$ & 6 & 0,28 & 0,945 \\
$\mathrm{R} \times \mathrm{H}$ & 6 & 1,19 & 0,313 \\
$\mathrm{C} \times \mathrm{H}$ & 12 & 0,76 & 0,693 \\
$\mathrm{P} \times \mathrm{R} \times \mathrm{C}$ & 2 & 2,10 & 0,126 \\
$\mathrm{P} \times \mathrm{R} \times \mathrm{H}$ & 6 & 0,12 & 0,993 \\
$\mathrm{P} \times \mathrm{C} \times \mathrm{H}$ & 12 & 0,75 & 0,704 \\
$\mathrm{R} \times \mathrm{C} \times \mathrm{H}$ & 12 & 0,52 & 0,902 \\
$\mathrm{P} \times \mathrm{R} \times \mathrm{C} \times \mathrm{H}$ & 12 & 0,16 & 0,999 \\
\hline
\end{tabular}

En la tabla 3 se observa que los factores Clase de copa y Altura relativa tienen un efecto altamente significativo sobre la variación de la densidad básica. En cambio, las intervenciones de Poda y Raleo no afectan en forma independiente a la variable de respuesta, sino que sólo en forma conjunta (interacción significativa).

En la tabla 4 se presentan los valores promedios de densidad básica de E. Nitens para todos los niveles de los diferentes factores estudiados. En ella se observa que, independiente del tratamiento silvícola aplicado a la unidad experimental, la densidad básica promedio por clase de copa siempre fue mayor en la dominante, seguido por la codominante y suprimida respectivamente. Cabe destacar que aquellos tratamientos silvícolas que incorporaron raleo $\left(\mathrm{P}_{0} \mathrm{R}_{800}\right.$ y $\left.\mathrm{P}_{60} \mathrm{R}_{800}\right)$ presentaron los mayores valores en densidad básica $\left(0,522 \mathrm{~g} / \mathrm{cm}^{3}\right.$ y $0,521 \mathrm{~g} / \mathrm{cm}^{3}$ respectivamente), independiente de su condición de poda (Tabla 4).

\section{Manejo silvícola}

En la figura 1 se observa el comportamiento de la densidad básica en función de la poda y el raleo, además de su interacción. La menor densidad básica se presentó en el tratamiento $\mathrm{P}_{60} \mathrm{R}_{1600}(0,487$ $\left.\mathrm{g} / \mathrm{cm}^{3}\right)$, siendo estadísticamente distinto a los tratamientos $P_{0} R_{1600}\left(0,506 \mathrm{~g} / \mathrm{cm}^{3}\right)$ y $P_{60} R_{800}\left(0,505 \mathrm{~g} / \mathrm{cm}^{3}\right)$, aunque estos no manifestaron diferencias significativas entre ellos.

De la figura 1 se deduce también que los tratamientos sin poda $\left(\mathrm{P}_{0}\right)$ presentan una mayor densidad promedio $\left(0,504 \mathrm{~g} / \mathrm{m}^{3}\right)$ que aquellos árboles que sí fueron sometidos a dicha intervención $\left(\mathrm{P}_{60}\right)$ 
$\left(0,496 \mathrm{~g} / \mathrm{cm}^{3}\right)$. De la misma forma, los tratamientos con raleo $\left(\mathrm{R}_{800}\right)$, presentaron una densidad básica promedio mayor $\left(0,503 \mathrm{~g} / \mathrm{m}^{3}\right)$ que aquellos tratamientos sin esta intervención $\left(\mathrm{R}_{1600}\right)\left(0,496 \mathrm{~g} / \mathrm{cm}^{3}\right)$. Aunque algunos trabajos en Eucalyptus grandis indican que la densidad de la madera generalmente incrementa o no se ve afectada por los tratamientos silviculturales (WILKINS, 1990; CROMER et al., 1996), otros comentan que la densidad básica incrementa al aumentar el espaciamiento en las plantaciones (WIKINS; KITAHARA, 1991; MALAN; HOON, 1992), existiendo una relación positiva entre los esfuerzos de crecimiento y la densidad básica (WILKINS; KITAHARA, 1991).

Tabla 4. Densidad básica $\left(\mathrm{g} / \mathrm{cm}^{3}\right)$ para cada nivel de los factores en estudio.

Table 4. Basic density $\left(\mathrm{g} / \mathrm{cm}^{3}\right)$ for every level of factors studied.

\begin{tabular}{lccccccccc}
\hline \multirow{2}{*}{ Tratamiento } & Clase de copa & DAT & DAP & ICM & Mitad & ACV & DCV & DLU & Promedio \\
\cline { 3 - 9 } & Suprimido & 0,467 & 0,425 & 0,463 & 0,496 & 0,483 & 0,512 & 0,530 & 0,482 \\
$\mathrm{P}_{0} \mathrm{R}_{800}$ & Codominante & 0,480 & 0,447 & 0,467 & 0,504 & 0,520 & 0,518 & 0,549 & 0,498 \\
& Dominante & 0,503 & 0,476 & 0,493 & 0,529 & 0,531 & 0,529 & 0,592 & $\mathbf{0 , 5 2 2}$ \\
\hline \multirow{3}{*}{$\mathrm{P}_{60} \mathrm{R}_{800}$} & Suprimido & 0,492 & 0,451 & 0,488 & 0,485 & 0,486 & 0,486 & 0,535 & 0,489 \\
& Codominante & 0,496 & 0,444 & 0,467 & 0,516 & 0,507 & 0,528 & 0,565 & 0,503 \\
& Dominante & 0,494 & 0,438 & 0,482 & 0,537 & 0,546 & 0,546 & 0,607 & $\boldsymbol{0 , 5 2 1}$ \\
\hline \multirow{3}{*}{$\mathrm{P}_{0} \mathrm{R}_{1600}$} & Suprimido & 0,479 & 0,457 & 0,470 & 0,504 & 0,539 & 0,549 & 0,542 & 0,506 \\
& Codominante & 0,476 & 0,462 & 0,474 & 0,517 & 0,530 & 0,530 & 0,547 & 0,505 \\
& Dominante & 0,489 & 0,475 & 0,487 & 0,515 & 0,509 & 0,525 & 0,549 & $\mathbf{0 , 5 0 7}$ \\
\hline \multirow{3}{*}{$\mathrm{P}_{60} \mathrm{R}_{1600}$} & Suprimido & 0,466 & 0,433 & 0,465 & 0,462 & 0,514 & 0,497 & 0,474 & 0,473 \\
& Codominante & 0,462 & 0,427 & 0,429 & 0,489 & 0,506 & 0,513 & 0,532 & 0,480 \\
& Dominante & 0,510 & 0,459 & 0,466 & 0,520 & 0,522 & 0,522 & 0,561 & $\boldsymbol{0 , 5 0 9}$ \\
\hline
\end{tabular}

DAT: Diámetro altura tocón; ACV: Antes de copa viva; DAP: Diámetro altura pecho; DCV: Después de copa viva; ICM: inicio de copa muerta; DLU: Diámetro límite de utilización $6 \mathrm{~cm}$; Mitad: Mitad del árbol.

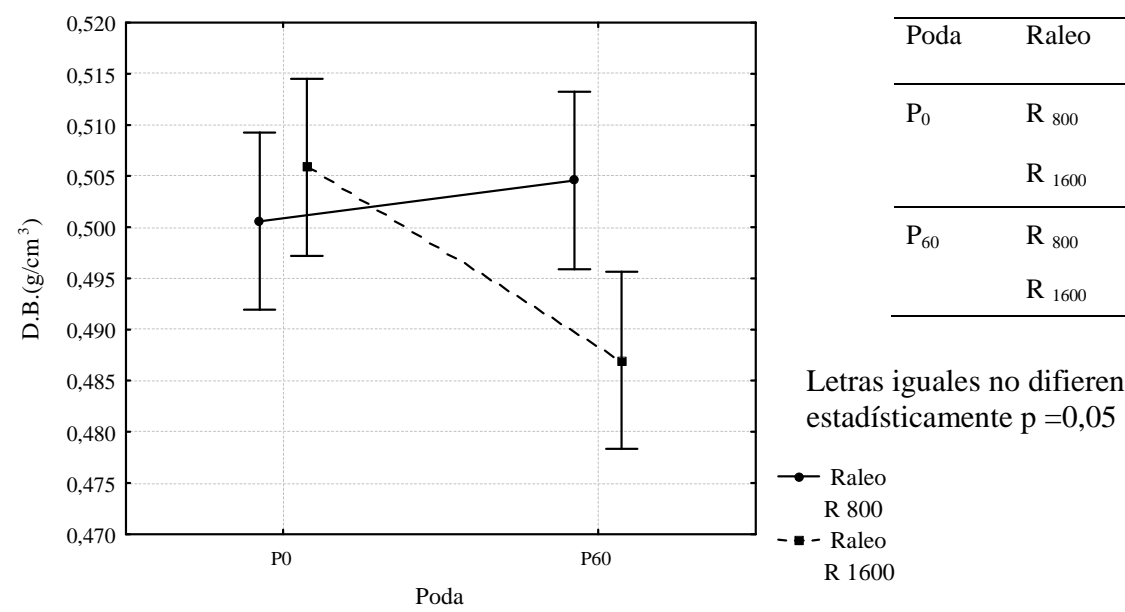

Figura 1. Densidad básica por poda y raleo.

Figure 1. Basic density by pruning and thinning.

\section{Clase de copa}

En la figura 2 se observa que los árboles de clase de copa dominante presentan la mayor densidad básica promedio $\left(0,515 \mathrm{~g} / \mathrm{cm}^{3}\right)$, siendo estadísticamente distinta a las clases de copa codominante $\left(0,496 \mathrm{~g} / \mathrm{cm}^{3}\right)$ y suprimido $\left(0,487 \mathrm{~g} / \mathrm{cm}^{3}\right)$ respectivamente, aunque estos dos últimos no difieren significativamente. La clase de copa dominante supera en un $3,7 \%$ a la clase codominante y en un $5,4 \%$ a la clase suprimida. 
Los resultados de este estudio demuestran la existencia de una relación proporcional entre la densidad básica de la madera y la vigorosidad de la copa, relación que se manifiesta en todas las condiciones de poda y raleo (Tabla 4).

Según Haygreen; Bowyer (1996) los árboles remanentes, después de raleos o cortas parciales, responden ante la mayor exposición al medio ambiente a través de la estimulación de sus copas. Por ende, son estimulados al aumentar la disponibilidad de espacio de crecimiento de cada uno de los árboles, al igual que la cantidad de luz y principalmente nutrientes, lo que equivale a aumentar el crecimiento de estos, vigorosidad de sus copas y simultáneamente su densidad básica.

Debido al impacto en el desarrollo de la copa y al ritmo de crecimiento, el raleo puede afectar adversamente algunas propiedades de la madera, sin embargo, los efectos pueden ser minimizados. Un raleo aplicado en forma temprana puede causar el mismo efecto que amplios espaciamientos de la plantación, uno de ellos es un incremento en el tamaño de la madera juvenil a través de mayores anchos de anillos de crecimientos en esta zona; además, puede provocar que este periodo se extienda, lo cual repercutirá en la densidad y resistencia y mayor contracción longitudinal al secarse (BERROCAL, 2001).

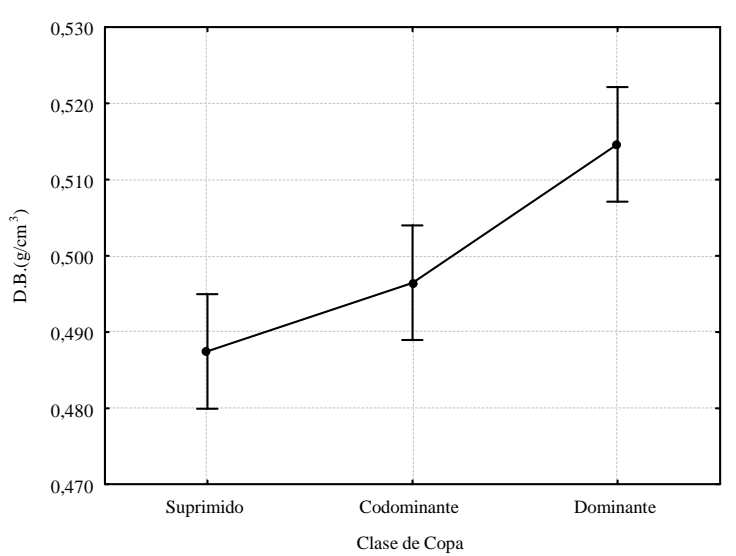

\begin{tabular}{lc}
\hline Clase Copa & $\begin{array}{c}\text { D. Básica } \\
\left(\mathrm{g} / \mathrm{cm}^{3}\right)\end{array}$ \\
\hline Suprimido & $0,487 \mathrm{a}$ \\
Codominante & $0,496 \mathrm{a}$ \\
Dominante & $0,515 \mathrm{~b}$ \\
\hline \multicolumn{2}{c}{ Letras iguales no difieren } \\
estadísticamente $\mathrm{p}=0,05$
\end{tabular}

Figura 2. Densidad básica por clase de copa.

Figure 2. Basic density by type of crown.

\section{Altura relativa}

En la figura 3 se grafica el comportamiento de la densidad de la madera en función de la altura relativa en el fuste, observándose claramente un aumento en la densidad básica desde la base al ápice. Si bien la densidad de la madera aumenta desde la base al ápice, esta variable presenta un decrecimiento a la altura del DAP e inicio de copa muerta (ICM), aunque esta disminución es estadísticamente significativa sólo en la primera sección citada (Figura 4 y Tabla 4). Desde la mitad del árbol, y hasta la sección correspondiente a después de copa viva (DCV), no se presentaron diferencias significativas; sin embargo, a la altura del DLU $6 \mathrm{~cm}$, la densidad básica fue estadísticamente distinta al resto de las secciones estudiadas.

Según Valencia; López (1999), el valor de la densidad de la madera y su variación, depende en alto grado de la altura y la sección del árbol de donde se toma la muestra. La tendencia más común observada para E. globulus es que la densidad básica aumenta con la altura (DEAN et al., 1990; VALENTE et al., 1992), acompañada algunas veces por una disminución en la base del árbol, al igual que en E. nitens (LLAUSBERG et al., 1995; BEADLE et al., 1996; RAYMOND; MACDONALD, 1998). Esta disminución inicial en la densidad de la madera es seguida por un incremento con la altura, observándose que la densidad mínima ocurre en la primera altura de muestreo, cerca de la base del árbol (BHAT et al., 1990; WILKINS, 1990; WILKINS; HORME, 1991; CLARK, 2001; KUBE; RAYMOND, 2002; KIBBLEWHITE et al., 2004). Raymond; MacDonald (1998) examinaron cambios en la densidad básica usando discos de madera del árbol en un rango de edades de 5, 10 y 15 años, en distintos sitios de Tasmania y Australia para E. globulus y E. nitens; ambas especies mostraron una disminución inicial de la densidad cerca de la base del árbol, seguida por un incremento lineal entre el $10 \%$ y $70 \%$ de la altura del árbol. 


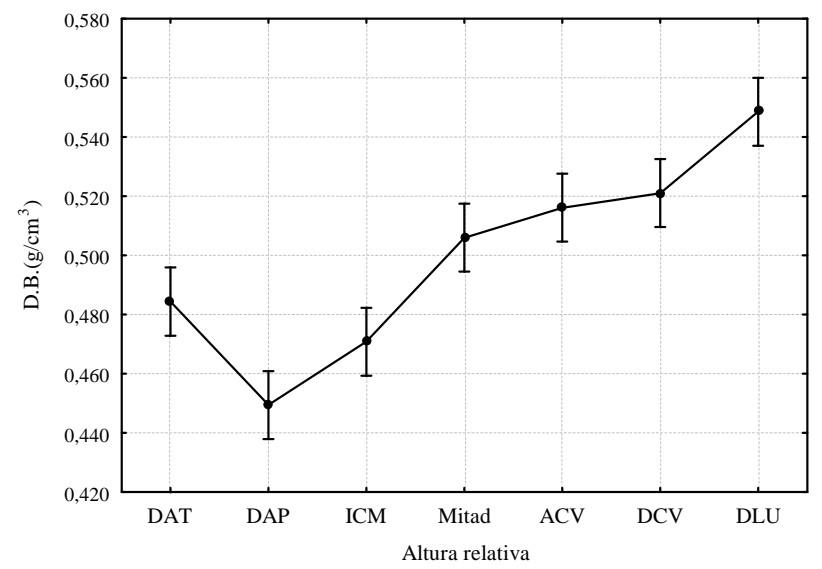

\begin{tabular}{lc}
\hline $\begin{array}{l}\text { Altura } \\
\text { relativa }\end{array}$ & $\begin{array}{c}\text { D. Básica } \\
\left(\mathrm{g} / \mathrm{cm}^{3}\right)\end{array}$ \\
\hline DAT & $0,484 \mathrm{bc}$ \\
DAP & $0,449 \mathrm{a}$ \\
ICM & $0,471 \mathrm{ab}$ \\
Mitad & $0,506 \mathrm{~cd}$ \\
ACV & $0,516 \mathrm{~d}$ \\
DCV & $0,521 \mathrm{~d}$ \\
DLU & $0,549 \mathrm{e}$ \\
\hline
\end{tabular}

Letras iguales no difieren estadísticamente $\mathrm{p}=0,05$

Figura 3. Densidad básica en función de la altura relativa en el árbol.

Figure 3. Basic density by relative height at tree.

\section{CONCLUSIONES}

De acuerdo a las condiciones en las que se realizó el presente estudio, y considerando los resultados obtenidos, se concluye que:

- El manejo silvícola afectó el comportamiento de la densidad de la madera, presentándose una mayor densidad básica promedio en las unidades experimentales con raleo y sin intervención de poda.

- A nivel de árbol individual, la altura relativa tuvo un efecto altamente significativo en la variación de la densidad básica, la cual tendió a aumentar desde la base al ápice, aunque presentó una leve disminución en las primeras secciones del fuste.

- La clase de copa dominante presentó una densidad básica superior a la codominante y suprimida, respectivamente.

\section{REFERENCIAS}

BALLONI, E. Spacing influences over Eucalyptus forest productivity. Silvicultura, São Paulo, ano 8, n. 31, p. 588-593, 1983. Simpósio Iufro em Melhoramento Genético e Productividade de Espésies Forestales de Rápido Crecimiento, 8., 1980, Águas de São Pedro, Brazil.

BAMBER, R.; BURLEY, J. The wood properties of Radiata pine. Slough: Common Wealth Agricultural Bureaux, 1983. 84 p.

BEADLE, C. L.; TURNBULL, C. R. A.; DEAN, G. H. Environmental effects on growth and kraft pulp yield of Eucalyptus globulus and Eucalyptus nitens. Appita, Victoria, n. 49, p.239-242, 1996.

BERROCAL, A. Efecto de las prácticas silviculturales en la estructura y morfología de la madera. Informe técnico. Concepción, Chile: Universidad de Concepción. Facultad de Ciencias Forestales, Programa de Postgrado,.2001.

BHAT, K.; DHAMODARAN, T. Wood density and fiber length of Eucalyptus grandis grown in Kerala, India. Wood and Fiber Science, Madison, n. 22, p. 54-61, 1990.

BRITO, C. Efecto del manejo temprano, en el crecimiento y dominancia de rodales jóvenes de Eucalyptus globulus y Eucalyptus nitens en un suelo precordillerano de la VIII Región. SEMINARIO DE TÍTULO INGENIERÍA DE EJECUCIÓN FORESTAL. Los Ángele: Universidad de Concepción. Unidad Académica. 2003. 60 p. 
CABRERA, J. Desarrollo de opciones productivas de mayor valor para plantaciones de Eucalyptus nitens en la $\quad$ IX $\quad y \quad r \quad X \quad$ región. $\quad$ Disponível <http://www.infor.cl/webinfor/investigacion/proyectos/eucanitens.html>. Acesso em: 2001.

CAMPOS, A.; CUBILlos, G.; MORAles, F.; PASTENE, A. Propiedades y usos de especies madereras de corta rotación. Santiago, Chile: CORFO-INFOR, 1990. 119 p.

CARRASCO, P.; MILLÁN, J.; ESPINOSA, M.; DRAKE, F. Suelos forestales de la VIII Región. Chillán, Chile: FIA/ Universidad de Concepción. Depto. Cs. Forestales, 1989.

CLARK, N. Longitudinal density variation in irrigated hardwoods. Appita, Victoria, v. 54, n. 1, p. 49-53, 2001.

CROMER, R.; BALODIS, B.; CAMERON, D.; GARLAND, C.; RANCE, S.; RYAN, P. Growth and pulping characteristics of Eucalyptus grandis in response to fertilizer Near Gympie, Queensland. In: APPITA ANNUAL GENERAL CONFERENCE, 50., 1996, Auckland, New Zealand. Proceedings of the... Auckland, 1996. p. 303-309.

DANIEL, P.; HELMS, U.; BAKER, F. Principios de silvicultura. Trad. Ramón Elizondo Mata. México: Mc Graw-Hill, 1982. 492 p.

DEAN, G.; FRENCH, J.; TIBBITS, W. Variation in pulp and papermaking characteristics in a field trial of Eucalyptus globulus. APPITA ANNUAL GENERAL CONFERENCE, 44., 1990, Rotorua. Proceedings of the... Rotorua, 1990.

FERNÁNDEZ-GOLFÍN, J.; DIEZ, M. Influencia del anillo de crecimiento en la densidad y otras propiedades físico-mecánicas de la madera estructural de diversas especies. Investigacion Agraria. Sistemas y Recursos Forestales, Madrid, v. 3, n. 2, p. 211-219, 1994.

GREAVES, B.; BORRALHO, N. The influence of basic density and pulp yield on the cost of eucalypt kraft pulping: a theoretical model for tree breeding. Appita, Rotorua. v. 49, n. 2, p. 90-96, 1996.

HAYGREEN, J.; BOWYER, J. Forest products and wood science. $2^{\text {nd }}$ ed. Ames: Iowa State University Pres, 1996.

HERNÁNDEZ, G. Proyecto análisis del secado y trabajabilidad de tres especies de Eucalyptus crecidas en Chile. Disponível em: 〈http://www.infor.cl/webinfor/investigacion/proyectos/6181.html> Acesso em: 2002.

INSTITUTO NACIONAL DE NORMALIZACIÓN (INN). Norma Chilena Oficial, NCh 176/1 Of84, "Madera-Determinación de Humedad". Primera Edición. Chile, 1984.

INSTITUTO NACIONAL DE NORMALIZACIÓN (INN). Norma Chilena Oficial, NCh 176/2 Of86, "Madera-Determinación de la Densidad". Chile, 1986.

ITURRIA, L. Crecimiento de un rodal de Pinus radiata D. Don en trumaos de la provincial del BíoBío sometido a manejo intensivo. Tesis de grado de Ingeniería Forestal. Universidad de Concepción. Facultad de Cs. Forestales. Concepción, Chile, 1997.

KIBBLEWHITE, R.; EVANS, R.; RIDDELL, M.; SHELBOURNE, C. Changes in density and woodfiber properties with height position in 15/16-year-old Eucalyptus nitens and Eycalyptus fastigata. Appita, Rotorua, v. 57, n. 3, p. 240-247, 2004.

KOLLMAN, F. Tecnología de la madera y sus aplicaciones. Madrid: Instituto Forestal de Investigación y Experiencias y Servicio de la Madera, 1959. 675 p.

KUBE, P.; RAYMOND, C. Prediction of whole-tree basic density and pulp yield using wood core samples in Eucalyptus nitens. Appita, Victoria, v. 55, n. 1, p. 43-48, 2002.

LLAUSBERG, M.; GILCHISST, K.; SKIPWITH, J. Wood properties of Eucalyptus nitens grown in New Zealand. New Zealand Journal of Forestry Science, Rotorua, v. 25, n. 2, p. 147-163, 1995. 
MALAN, F.; HOON, M. Effect of initial spacing and thinning on some wood properties of Eucalyptus grandis. South African Forestry Journal, Pretoria, v. 63, p. 13-20, 1992.

MONTGOMERY, D. Diseño y análisis de experimentos. México: Grupo Iberoamericano. 1991. 589 p.

PRADO, J.; BARROS, S. Eucalyptus: principios de silvicultura y manejo. Santiago, Chile: Impresos Nova, 1989.

RAYMOND, C.; McDONALD, A. Where to shoot pilodyn: within tree variation in basic density in plantation Eucalyptus globulus and Eucalyptus nitens in Tasmania. New Forests, Dordrecht, n. 15, p. 205-221, 1998

ROZENBERG, P.; FRANC, A.; BASTIEN, C.; CAHALAN, C. Improving models of wood density by including genetics effects: A cause study in Douglas-fir. Annals of Forest Science, Versailles, v. 58, p. 385-394, 2001.

TIAN, X.; COWN, D.; McCONCHIE, D. Modelling of Pinus radiata wood properties. Part 2: Basic density. New Zealand Journal of Forestry Science, Rotorua, v. 25, n. 2, p. 214-230, 1995.

USTA, I.; HALE, M. Effects of seed origin and site on wood density of Sitka spruce (Picea sitchensis (Bong.) Carr.) grown in Britain. Turkish Journal of Agriculture and Forestry, Ankara, v. 26, p. 21-30, 2002.

VALENCIA, J.; CABRERA, J. Plantaciones de Eucalyptus nitens como opción forestal de alto valor. SEMINARIO INVESTIGACIÓN Y DESARROLLO FORESTAL EN LA PEQUEÑA PROPIEDAD, 2., Concepción, 2003. Resúmenes de... Concepción, Chile: INFOR, 2003.

VALENCIA, M.; LÓPEZ, F. Variación de la densidad de la madera dentro y entre árboles de Pinus rudis Endl., en Sierra Las Alazanas, Artega, Coah. Foresta-An. Saltillo, Coah: UAAAN, 1999. 17 p. (Nota Técnica, n. 1).

VALENTE, C.; MENDES DE SOUSA, A.; FURTADO, F.; CARVALHO, A. Improvement program for Eucalyptus globulus at Portucel: Technological component. Appita, Victoria, n. 45, p. 403-407, 1992.

WILKINS, A. Influence of silvicultural treatment on growth and wood density of Eucalyptus grandis grown on a previous pasture site. Australian Forestry, Yarralumla, v. 53, n. 3, p. 168-172, 1990.

WILKINS, A.; HORNE, R. Wood-density variation of young plantation-grown Eucalyptus grandis in response to silvicultural treatments. Forest Ecology and Management, Amsterdam, v. 40, p. 39-50, 1991.

WILKINS, A.; KITAHARA, R. Silvicultural treatments an associated growth rates, growth strains and wood properties in 12,5 year-old Eucalyptus grandis. Australian Forestry, Yarralumla, v. 54, p. 99-104, 1991.

ZOBEL, B.; TALBERT, J. Técnicas de mejoramiento genético de árboles forestales. México: Limusa, $1988.545 \mathrm{p}$. 